\title{
Efficacy and safety of venetoclax in combination therapy for relapsed acute myeloid leukemia after allogeneic hematopoietic stem cell transplantation
}

\author{
Fan Yang, Yueqian Feng, Changwei Dong, Zhihui Li, Tong Wu \\ Department of Bone Marrow Transplantation, Beijing Boren Hospital, Beijing, China
}

\begin{abstract}
Objective: To evaluate the efficacy of the B-cell lymphoma 2 (BCL2)-inhibitor venetoclax in combination therapy for relapsed acute myeloid leukemia (AML) patients after allogeneic hematopoietic stem cell transplantation (allo-HSCT).

Methods: We retrospectively analyzed 7 adults with relapsed AML who failed at least one prior therapy and were treated with venetoclax in combination with decitabine and/or low-dose cytarabine at the Beijing Boren hospital between March 2019 and March 2020. Four patients (57.1\%) had adverse cytogenetic findings. Four patients $(57.1 \%)$ had undergone a donor lymphocyte infusion (DLI) prior to venetoclax therapy, while four patients $(57.1 \%)$ had leukemia and failed prior hypomethylating agent (HMA) therapy.

Results: The objective response rate (ORR) was $71.4 \%$ (5 patients achieved morphological complete remission with incomplete hematologic recovery, CRi). At the end of the follow-up period, 3/5 cases $(60 \%)$ in the CRi group achieved minimal residual disease (MRD) negativity by flow cytometry. One patient (14.3\%) successfully underwent allo-HSCT. The median follow-up time was $140(120,354) \mathrm{d}$. Among the seven patients, one died of relapse after remission, with an overall survival rate of $85.7 \%$ and a disease-free survival rate of $57.1 \%$ (4/7). Five of these patients $(71.4 \%)$ had an identifiable infection, including septicemia (one patient), herpes zoster (two patients), and pneumonia (two patients). One patient had a tumor lysis syndrome.

Conclusion: The venetoclax-based combinations demonstrated efficacy in treating adult patients with AML relapsing after allo-HSCT.
\end{abstract}

Key words acute myeloid leukemia, allogeneic hematopoietic stem cell transplantation, relapse, venetoclax

Submitted December 31, 2020; Accepted March 20, 2021; Published online August 25, 2021; Issued online August 25, 2021

Correspondence: Tong Wu, Department of Bone Marrow Transplantation, Beijing Boren Hospital, Block A, Longwei Plaza, Jitong East Road, Fengtai District, Beijing, 100070, China, E-mail: wut@borenhospital.com

\section{Introduction}

Acute myeloid leukemia (AML) relapse remains the leading cause of treatment failure after allogeneic hematopoietic stem cell transplantation (allo-HSCT) with a two-year survival rate of $<20 \%$, despite various strategies including hypomethylating agents, chemotherapy, donor lymphocyte infusion (DLI), target drugs, and secondary transplantation ${ }^{1}$. Recent studies have shown that the anti-apoptotic protein B-cell lymphoma 2 (BCL-2) overexpression of leukemic cells can prevent tumor cell apoptosis, shorten the overall survival of AML patients, and cause resistance to therapy ${ }^{1}$. Venetoclax is a highly selective inhibitor of BCL-2 that can directly bind to the BCL-2 protein to substitute a pro-apoptotic protein and restart the process of apoptosis. Venetoclax combined with hypomethylating agents (HMAs) and lowdose cytarabine has demonstrated much better responses in relapsed $\mathrm{AML}^{2}$. However, there are limited data on the efficacy of venetoclax in relapsed AML after alloHSCT. Our study aimed to provide evidence for the efficacy, toxicity, and response characteristics of venetoclax combination therapy in relapsed AML after alloHSCT.

\section{Patients and Methods}

\section{Cases}

A retrospective analysis was performed on seven patients with relapsed AML after allo-HSCT treated with 
Table 1. Patient and Characteristics at Time of Venetoclax Combination Therapy

\begin{tabular}{|c|c|c|c|c|c|c|c|}
\hline & $\# 1$ & $\# 2$ & $\# 3$ & $\# 4$ & $\# 5$ & $\# 6$ & $\# 7$ \\
\hline Age & 27 & 52 & 52 & 47 & 38 & 16 & 43 \\
\hline Sex & Female & Female & Female & Female & Female & Male & Female \\
\hline Diagnosis & De novo & Secondary & Secondary & De novo & De novo & De novo & De novo \\
\hline Cytogenetics & Adverse & Unknown & Unknown & Adverse & Adverse & Adverse & Intermediate \\
\hline Chimerism & $\begin{array}{l}\text { t (8; 21), } \\
t(3 ; 12),-x\end{array}$ & Unknown & Unknown & $\operatorname{inv}(3),-7$ & $\begin{array}{c}1 p+,+4, \text { dre (13), } \\
16 p+,-18,-20,21 p+\end{array}$ & Unknown & Unknown \\
\hline Transplant donor & MRD & MRD & Haplo & Haplo & MRD & MUD & Haplo \\
\hline Conditioning regimens & FB2 & $\mathrm{Cy}+\mathrm{FB} 3$ & FB3 & FB4 & $\mathrm{Dec} / \mathrm{Bu} / \mathrm{Cy}$ & $\mathrm{Bu} / \mathrm{Cy}$ & $\mathrm{Bu} / \mathrm{Cy}$ \\
\hline aGvHD & None & None & Unknown & 3 & None & None & 1 \\
\hline cGvHD & None & None & None & Extensive & None & None & None \\
\hline Relapsing time post-HSCT & +44 & +467 & +49 & +116 & +364 & +29 & +27 \\
\hline Hypomethylating agents & No & Yes & Yes & Yes & Yes & No & No \\
\hline DLI & Yes & Yes & No & Yes & Yes & No & No \\
\hline Bone marrow morphology & $34 \%$ & $18 \%$ & $13 \%$ & $0.5 \%$ & $51 \%$ & $8 \%$ & $60 \%$ \\
\hline Mutations & None & Unknown & MPL, JAK2 & Unknown & TP53 & None & NPM, FLT3 \\
\hline MRD by flow cytometry & $9.09 \%$ & $8.02 \%$ & $13.8 \%$ & $0.03 \%$ & $45.3 \%$ & $22.92 \%$ & $20 \%$ \\
\hline
\end{tabular}

Cy, cyclophosphamide; Bu/Cy, busulfan/cyclophosphamide; FB2, fludarabine + busulfan (2 days); FB3, fludarabine + busulfan (3 days); FB4, fludarabine + busulfan (4 days); DLI, donor lymphocyte infusion; MRD, match related donor; MUD, match unrelated donor; haplo, related haplotype donor; aGvHD, acute graft-versus-host disease; cGvHD, chronic graft-versus-host disease; MRD by Flow cytometry, Microresidual disease by Flow cytometry

venetoclax-based combination therapy at Beijing Boren hospital from March 1, 2019 to March 1, 2020. Disease characteristics of these patients are shown in Table 1. The median age was 43 y (range, 16-52), with only one male patient $(14.3 \%)$. The median time from relapsing post-HSCT to venetoclax combination therapy was 49 days (range, 27-467). Five patients $(71.4 \%)$ had de novo AML, and two patients (28.6\%) had AML with myelodysplastic changes. Four patients $(57.1 \%)$ had leukemia characterized by adverse cytogenetics and one by intermediate cytogenetics. Notable molecular mutations present were tumor protein 53 (TP53, one patient, 14.3\%), fms-like tyrosine kinase 3 (FLT3, one patient, 14.3\%), nucleophosmin 1 (NPM1, 1 patient, 14.3\%), Janus tyrosine kinase 2 (JAK2, one patient, 14.3\%), and MPL (one patient, 14.3\%). Six patients (85.7\%) were treated with chemotherapy and/or HMAs, except for one patient who was untreated before initiation of venetoclax. Four patients (57.14\%) had received DLI prior to venetoclax therapy, and six patients $(85.7 \%)$ had leukemia that failed prior therapy. One patient $(14.3 \%)$ had grade 3 aGvHD prior to starting venetoclax therapy, and one patient (14.3\%) had extensive type cGvHD. The median Eastern Cooperative Oncology Group (ECOG) score was 1 (range 0-2) for patients. The study was conducted in accordance with accepted ethical guidelines and received approval from the hospital' s institutional review board, and all patients provided written informed consent to participate.

\section{Medication method}

Five patients received venetoclax in combination with low-dose cytarabine (LDAC) $\left(20 \mathrm{mg} / \mathrm{m}^{2} \times 14 \mathrm{~d}\right)$, while two patients received venetoclax with decitabine (15 $\left.\mathrm{mg} / \mathrm{m}^{2} \times 5 \mathrm{~d}\right)$ and LDAC $\left(20 \mathrm{mg} / \mathrm{m}^{2} \times 14 \mathrm{~d}\right)$. All the patients received venetoclax dosing as a "ramp-up" (starting at a lower dose and then increasing it when tolerated). The dosing of venetoclax was at the physician's discretion with a starting dose of 100 or $200 \mathrm{mg} / \mathrm{d}$. The dose was gradually increased to the maximum of 600 $\mathrm{mg} / \mathrm{d}$, with the administration lasting for a total of 28 days if the patient showed satisfactory tolerance. When the neutrophil count was $\leq 0.5 \times 10^{9} / \mathrm{L}$, venetoclax therapy was stopped and granulocyte colony stimulating factor (G-CSF) was administered during treatment. Venetoclax monotherapy $(400 \mathrm{mg} \times 28 \mathrm{~d}$ ) or combination therapy such as Venetoclax $(400 \mathrm{mg} \times 28 \mathrm{~d})$ and LDAC $\left(20 \mathrm{mg} / \mathrm{m}^{2} \times 14\right.$ days $)$ or decitabine $\left(15 \mathrm{mg} / \mathrm{m}^{2} \times 5 \mathrm{~d}\right)$ would be administered every two months if they led to better responses. (Because it is a retrospective study, the scheme is in the exploratory stage, so there is no unified standard).

\section{Efficacy criteria and safety evaluation}

Using the Hematological Diagnosis and Efficacy Criteria $^{3}$, medical records were retrospectively analyzed for disease characteristics, laboratory values, number and type of prior therapies, cytogenetics, molecular mutations, characteristics, and response to venetoclax combination therapy, side effects of venetoclax combination 
Table 2. Patients' characteristics and outcomes $(n=7)$

\begin{tabular}{|c|c|c|c|c|c|c|c|}
\hline & $\# 1$ & \#2 & \#3 & $\# 4$ & $\# 5$ & $\# 6$ & $\# 7$ \\
\hline $\begin{array}{l}\text { Venetoclax maximum dose } \\
\text { for one day }(\mathrm{mg})\end{array}$ & 400 & 600 & 400 & 400 & 400 & 600 & 600 \\
\hline Combination agents & Cytarabine & Cytarabine & $\begin{array}{l}\text { Cytarabine/ } \\
\text { Decitabine }\end{array}$ & Cytarabine & $\begin{array}{l}\text { Cytarabine/ } \\
\text { Decitabine }\end{array}$ & Cytarabine & Cytarabine \\
\hline Best response & CRi & CRi & CRi & CRi & CRi & NR & NR \\
\hline $\begin{array}{l}\text { Number of venetoclax cycle } \\
\text { to achieve best response }\end{array}$ & 1 & 1 & 1 & 1 & 1 & - & - \\
\hline MRD by flow cytometry & $0 \%$ & $0 \%$ & $3.21 \%$ & $0 \%$ & $12.9 \%$ & $8.76 \%$ & $47.32 \%$ \\
\hline $\begin{array}{l}\text { Donor implantation rate } \\
\text { after best response }\end{array}$ & $100 \%$ & $100 \%$ & $100 \%$ & $100 \%$ & $100 \%$ & $47.12 \%$ & $55 \%$ \\
\hline $\begin{array}{l}\text { Number of venetoclax } \\
\text { cycles as Maintenance }\end{array}$ & 2 & 2 & 0 & 4 & 2 & 0 & 0 \\
\hline Maintenance agents & $\begin{array}{l}\text { Venetoclax+ } \\
\text { Cytarabine }\end{array}$ & Venetoclax & none & $\begin{array}{l}\text { Venetoclax+ } \\
\text { Decitabine }\end{array}$ & $\begin{array}{l}\text { Venetoclax+ } \\
\text { Cytarabine }\end{array}$ & CART & Sorafenib \\
\hline Secondary HSCT & No & No & Yes & No & No & No & No \\
\hline Current status & $\begin{array}{l}\text { Alive without } \\
\text { leukemia }\end{array}$ & $\begin{array}{l}\text { Died from } \\
\text { relapse }\end{array}$ & $\begin{array}{l}\text { Alive without } \\
\text { leukemia }\end{array}$ & $\begin{array}{l}\text { Alive without } \\
\text { leukemia }\end{array}$ & $\begin{array}{l}\text { Alive without } \\
\text { leukemia }\end{array}$ & $\begin{array}{l}\text { Alive with } \\
\text { leukemia }\end{array}$ & $\begin{array}{l}\text { Alive with } \\
\text { leukemia }\end{array}$ \\
\hline Survival (days) & 147 & 241 & 205 & 352 & 120 & 210 & 202 \\
\hline
\end{tabular}

$\mathrm{CR} / \mathrm{CRi}$, complete remission/complete remission with incomplete hematologic recovery; NR, no remission; CART, Chimeric antigen receptor T cells; MRD by flow cytometry, Microresidual disease by flow cytometry

therapy, and survival. Cytogenetic and molecular risk were classified according to the 2017 European leukemia net (ELN) risk stratification, and response to venetoclax combination therapy was assessed according to the 2017 ELN response criteria ${ }^{4}$. All the patients were intermittently examined for coagulation function, routine blood parameters, liver function, renal function, and cardiac function after treatment. Complications were recorded in detail.

\section{Results and Discussion}

Poor treatment results in AML relapse after HSCT led to a change in our practice to include venetoclaxbased therapy in failure strategies. A retrospective analysis by Aldoss et al. of 33 relapsed AML patients treated with venetoclax combination therapy revealed that 6 of the $13(46.2 \%)$ enrolled patients relapsed after allo-HSCT showed efficacy 5 . We found an ORR of $71.4 \%$ in our patients, with five patients achieving $\mathrm{CRi}$, of which three out of five (60\%) achieved MRD negativity by flow cytometry, including two patients who received combination of low-dose cytarabine and decitabine. The characteristics and outcomes of patients are shown in Table 2. The median time to the best response was 34 days (range, 24-48) for one cycle. There was no difference in the dosage of venetoclax combination between the CRi and NR groups. Similar to another study ${ }^{6}$, all CRi patients were treated with HMAs and/or DLI before venetoclax combination therapy. One patient was able to use venetoclax combination therapy as an abridge to secondary allo-HSCT. Three cases were effective and all survived without leukemia; however, one patient relapsed after single venetoclax maintenance during the median follow-up time of 205 days (range 120-352). In the NR group, two patients with leukemia survived. Six patients survived during the follow-up period, with a total survival rate of $85.7 \%$. Our data showed that combining HMAs with venetoclax showed more encouraging anti-leukemia activity, higher response rate, and greater overall survival in AML patients who relapsed after allo-HSCT.

We also observed an encouraging response in patients with adverse cytogenetic findings. In our population, one patient with TP53 mutation cleared the mutation with venetoclax combined with low-dose cytarabine and decitabine and survived free of leukemia up to the follow-up period. Although AML patients with TP53 mutation may be more sensitive to decitabine ${ }^{6}$, early work indicates these patients may derive less benefit from venetoclax? Additional studies on the mechanism of venetoclax combined with low-dose cytarabine and decitabine in patients with TP53 mutations are needed in large-scale case analysis.

Some data suggest that venetoclax combined with FLT3 inhibitors is effective in treating FLT3-mutated AML. Similar to other retrospective analyses of venetoclax combination therapy in relapsed AML, three out of four AML patients with FLT3-mutation had responses, and two of the three patients with FLT3-mutation were concurrently treated with sorafenib ${ }^{8}$. We suggest that the FLT3 inhibitor may raise the possibility of a synergistic effect of the two drugs, although the patient with FLT3mutated in in our study did not respond to venetoclax 
combination sequential sorafenib. Therefore, simultaneous targeting of BCL-2 and FLT3 may be a way to overcome primary and secondary resistance in AML patients receiving venetoclax therapy.

The most common and serious adverse reaction to venetoclax is neutropenia, which generally occurs at the beginning of treatment ${ }^{9}$. Other side effects $(>30 \%)$ include nausea, diarrhea, constipation, febrile neutropenia, fatigue, hypokalemia, loss of appetite, and leukopenia. In our study, five patients $(71.4 \%)$ experienced neutropenia during the first cycle. The median time from venetoclax initiation to neutropenia in patients with $\mathrm{CRi}$ was 14 days which was evidently longer than the 1.5 days in NR group. Compared with CRi group, the median duration of grade 3 or greater neutropenia was much longer in the NR group. Five out of seven patients $(71.4 \%)$ had an identifiable infection including septicemia (one patient), herpes zoster (two patients), and pneumonia (two patients), and one patient showed tumor lysis syndrome. Gastrointestinal side effects were common: seven patients $(100.0 \%)$ had nausea and three patients $(42.8 \%)$ had vomiting. Four patients $(57.1 \%)$ developed hyperbilirubinemia, which improved with drug withdrawal. In dose climbing trials for AML patients, the initial dose of venetoclax was not less than $200 \mathrm{mg} / \mathrm{d}$, with no reported tumor lysis syndrome. Nevertheless, complications have been reported in patients with chronic lymphocytic leukemia (CLL), which is attributed to the breakdown and death of a large number of tumor cells because of the rapid onset of veneto$\operatorname{clax}^{10}$. For this reason, CLL patients are recommended to start with a small dose, such as $10 \mathrm{mg} / \mathrm{d}$. Given that our patient experienced tumor lysis syndrome upon administration of $200 \mathrm{mg}$, a large sample size is needed to confirm whether to further reduce the initial dose of venetoclax for relapsed AML patients after transplantation.

In summary, the response rates and survival data of venetoclax combination therapy in relapsed AML patients after allo-HSCT indicate improved outcomes and can become a bridging treatment for secondary alloHSCT.

\section{Acknowledgments}

The authors would like to thank Dong Chen, the division of Hematopahtology, Mayo Clinic, Rochester, $\mathrm{MN}$ for his proofreading to publish this article.

\section{Author Contributions}

YF, CD, and ZL implemented the clinical protocol,
TW designed the research.

\section{Conflicts of Interest}

The authors declare no conflict of interest. Disclosure forms provided by the authors are available on the website.

\section{References}

1. Soiffer RJ, Chen YB. Pharmacologic agents to prevent and treat relapse after allogeneic hematopoietic cell transplantation. Blood Adv. 2017; 1: 2473-82.

2. DiNardo CD, Pollyea DA, Jonas BA, Konopleva M, Pullarkat V, Wei A, et al. Updated Safety and Efficacy of venetoclax with decitabine or azacitidine in Treatment-Naive, Elderly Patients with Acute Myeloid leukemia. Blood. 2017; 130 (Supplement 1): 2628.

3. Shen T, Zhao YQ. Diagnostic and Efficacy Standards for Hematological Diseases, 4th ed. Science Press, 2018, p. 97-110.

4. Döhner H, Estey E, Grimwade D, Amadori S, Appelbaum FR, Büchner T, et al. Diagnosis and management of AML in adults: 2017 ELN recommendations from an international expert panel. Blood. 2017; 129: 424-47.

5. Aldoss I, Dongyun Y, Aribi A, Ali H, Sandhu K, Malki MMA, et al. Efficacy of the combination of venetoclax and hypomethylating agents in relapsed/refractory acute myeloid leukemia. Haematologica. 2018; 103: e404-7.

6. Welch JS, Petti AA, Miller CA, Fronick CC, O'Laughlin M, Fulton RS, et al. TP53 and decitabine in acute myeloid leukemia and myelodysplastic syndromes. N Engl J Med. 2016; 375: 2023-36.

7. DiNardo CD, Pratz K, Pullarkat V, Jonas BA, Arellano M, Becker PS, et al. Venetoclax combined with decitabine or azacitidine in treatment-naive, elderly patients with acute myeloid leukemia. Blood. 2019; 133: 7-17.

8. Gauta D, Burkenroada A, Duongb T, Feammelli J, Sasine J, Schiller G. Venetoclax combination therapy in relapsed/refractory acute myeloid leukemia: A single institution experience. Leuk Res. 2020; 90: 106314.

9. Konopleva M, Pollyea DA, Potluri J, Chyla B, Hogdal L, Busman T, et al. Efficacy and biological correlates of response in a phase II study of venetoclax monotherapy in patients with acute myelogenous leukemia. Cancer Discov. 2016; 6: 1106-17.

10. Roberts AW, Davids MS, Pagel JM, Kahl BS, Puvvada SD, Gerecitano JF, et al. Targeting BCL2 with venetoclax in relapsed chronic lymphocytic leukemia. N Engl J Med. 2016; 374: $311-22$.

https://doi.org/10.31547/bct-2020-025

Copyright (C)2021 Asia-Pacific Blood and Marrow Transplantation Group (APBMT). This is an open access article distributed under CC BY-NC license (https://creativecommon s.org/licenses/by-nc/4.0/). 